\title{
Massive hemothorax immediately after removal of central venous catheter
}

\section{-A case report-}

\author{
Hae-Kyoung Lee ${ }^{1}$, Seong-Wook Hong ${ }^{1}$, Gun-Jik Kim² ${ }^{2}$ Taeha Ryu ${ }^{3}$, Jae-Kyung Han ${ }^{1}$, and Jong-Chan Kim ${ }^{1}$ \\ ${ }^{1}$ Department of Anesthesiology and Pain Medicine, ${ }^{2}$ Division of Cardiovascular Surgery, Kyungpook National University Hospital, \\ ${ }^{3}$ Department of Anesthesiology and Pain Medicine, School of Medicine, Catholic University of Daegu, Daegu, Korea
}

Hemothorax is a possible immediate complication of central venous catheterization. We experienced a patient who suffered from massive hemothorax 72 hours after right subclavian venous catheterization. A 29-year-old female patient with Marfan's syndrome underwent the Bentall's operation and aortic arch replacement with an artificial graft, which was performed uneventfully. She recovered favorably in the intensive care unit and was transferred to the general ward on postoperative day 3. Immediately after the removal of the catheter in the general ward, massive hemothorax developed and emergent thoracotomy should have been performed to control bleeding. We report this case to re-emphasize the careful monitoring even after removal of central venous catheter and the need for ultrasound guidance during insertion of central venous catheters. (Korean J Anesthesiol 2013; 65: 77-79)

Key Words: Central venous catheterization, Patient safety, Hemothorax.

Central venous catheter placement for hemodynamic monitoring including central venous pressure (CVP) has become a clinically common and useful procedure in patients undergoing major surgery. Although the cannulation of the subclavian vein by the infraclavicular approach has advantages including relatively easy cannulation, comfort for patients and lower rate of infection, it is more frequently associated with mechanical complications such as pneumothorax, hemothorax and cardiac tamponade than other methods for access to central veins [1], which are mainly considered immediate complications [2]. However, we experienced a patient who suffered from hemo- thorax 72 hours following the procedure.

Herein we report a case of massive hemothorax due to hemorrhage immediately after removal of central venous catheter placed using infraclavicular approach.

\section{Case Report}

A 29-year-old woman with Marfan's syndrome was scheduled to undergo the elective Bentall's operation and thoracic aortic replacement with artificial graft. There were no abnormal findings or special histories on the preoperative evaluations.

Received: July 24, 2012. Revised: 1st, August 23, 2012; 2nd, September 12, 2012. Accepted: September 12, 2012.

Corresponding author: Jong-Chan Kim, M.D., Department of Anesthesiology and Pain Medicine, Kyungpook National University Hospital, 50, Samduk-2ga, Chung-gu, Daegu 700-721, Korea. Tel: 82-53-200-5863, Fax: 82-53-426-2760, E-mail: aescula72@hanmail.net

(c) This is an open-access article distributed under the terms of the Creative Commons Attribution Non-Commercial License (http:// creativecommons.org/licenses/by-nc/3.0/), which permits unrestricted non-commercial use, distribution, and reproduction in any medium, provided the original work is properly cited. 
On patient's arrival at the operating room, standard monitoring devices were applied including the left radial arterial cannulation. Anesthesia was induced and maintained according to institutional guidelines. After anesthetic induction, the right subclavian vein was cannulated with $\mathrm{MAC}^{\mathrm{TM}} \mathrm{Multi}$ Access Catheter (ARROW international Inc., PA, USA) using the infraclavicular Seldinger technique. During this attempt to cannulate the subclavian vein, there was neither air aspiration nor arterial puncture. After ensuring the aspiration of dark and nonpulsatile blood, a guidewire was introduced without any resistance. This was followed by sliding the catheter over the guidewire, and the wire was withdrawn without apparent resistance. Thus, the catheter was placed without any difficulty during catheterization. After that, the patient received standardized management according to institutional guidelines including mechanical ventilation and cardiopulmonary bypass during intraoperative period.

The operation was performed uneventfully. The patient was transferred to the intensive care unit (ICU) and was very stable in vital signs and laboratory tests. On postoperative day 1 , the patient was successfully weaned from mechanical ventilation. There was no evidence of hemorrhage including the volume of blood drained to chest bottles and follow-up chest X-ray (Fig. 1). On postoperative day 3 , the patient was transferred to the general ward and the central venous catheter was removed. Ten minutes after the removal of the catheter, the patient complained of dyspnea and pleuritic pain on right side, at which time approximately $1,700 \mathrm{ml}$ of blood was abruptly drained to chest bottles and the patient's chest X-ray confirmed a hemothorax on the same side as the cannulation. Although the blood

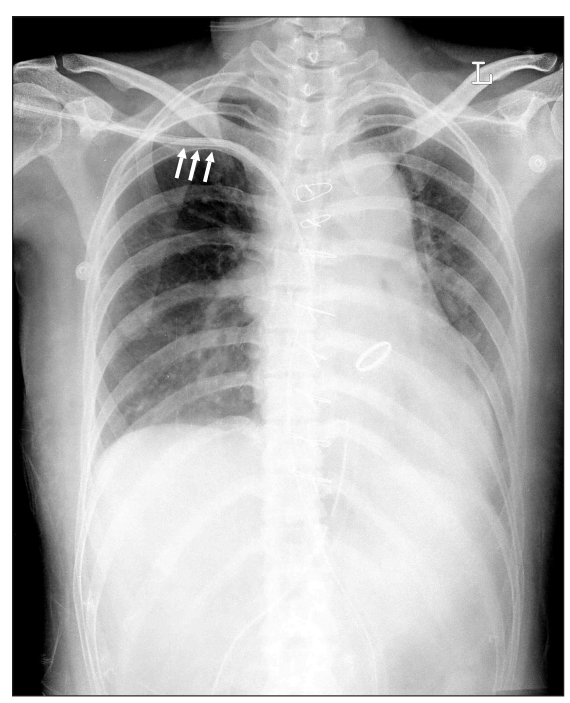

Fig. 1. Chest X-ray on postoperative day 1 . The catheter is parallel to the wall of right subclavian vein and shows a gentle curvature within the superior vena cava (Arrows). pressure was stable with administration of intravenous fluid and norepinephrine, hematocrit values dropped from $32.0 \%$ to $22.6 \%$.

The patient promptly underwent emergent thoracotomy and approximately 2,500 $\mathrm{ml}$ of clotted blood was evacuated during operation. The patient was maintained hemodynamically stable with infusion of $0.05 \mathrm{mcg} / \mathrm{kg} / \mathrm{min}$ of norepinephrine, $3,500 \mathrm{ml}$ of crystalloid and 4 units of packed red blood cell. There was an injury site by the central venous catheterization on the right side of superior vena cava, which was $3 \mathrm{~cm}$ distant from the junction of the right atrium and superior vena cava without any other injuries and reinforced by hemostatic materials. After transfer to the ICU, vital signs and other laboratory findings such as arterial blood gas analysis were stable without any pharmacologic supports. The patient was transferred to the general ward after a 4-day ICU stay and discharged on the 15th day after the thoracotomy without any complications.

\section{Discussion}

Central venous catheterization has many important roles in anesthesia and ICU care and can be conducted in central veins such as internal jugular, subclavian and femoral veins. There have been several reports about the advantages and disadvantages of each route. Although subclavian vein cannulation has been chosen for several advantages such as lower rate of infectious and thrombotic complications, stability, wide diameter of vessel and constant anatomical relationship, it is more frequently associated with mechanical complications including pneumothorax and hemothorax than other methods for access to central veins [1]. Complications of central venous catheterization involve immediate and late complications. The former is mainly associated with mechanical complications and the latter results from infection of the catheter [2].

The risk factors for mechanical complications and failures of subclavian vein catheterization include body-mass index (higher than 30 or lower than 20), women, previous catheterization, previous major surgery or radiation therapy in the region, only one year of training, and more than two needle passages [3]. In this case, however, the patient had no risk factors except woman. Also, there have been several reports about early hemorrhagic complications associated with subclavian venous catheterization. S. Holt and colleagues reported a hemothorax due to pulmonary arterial bleeding within 10 minutes after supraclavicular approach to subclavian vein [4]. Two cases were reported about hemothorax after removal of subclavian vein catheter, which described hemothorax in a child (age 13 months) [5] and hemothorax due to ipsilateral arteriovenous fistula for hemodialysis after removal of subclavian vein catheter in adult [6]. In this case, there were no symptoms or 
evidence for hemothorax including laboratory test, drained blood volume and vital signs until the catheter was removed on postoperative day 3. Although the reason of late hemorrhagic complications remains unclear, some reasons have been suggested including the pleural injury during catheterization, the erosion of the vessel wall due to catheter itself and the infusion of hyperosmolar solution [6-8]. However, in our case, these reasons do not seem likely, because the hemothorax was developed within 10 minutes after removal of the catheter. Some cases have been reported about perforation of vena cava or heart causing hemothorax despite of adequate direction and length of needle insertion because of contraction of the heart and stiffness of catheter [9]. In addition, it was reported that superior vena cava was displaced to the right by dissecting aorta [10]. Accordingly, we assume that although the subclavian catheter was misplaced and injured to right-shifted superior vena cava directly, the tip of catheter fit the injured hole perfectly, which made the patient asymptomatic and made physicians certain that the catheterization was conducted well. To our knowledge, it is very rare that the adult patient who was very stable hemodynamically and had no symptoms and clinical evidences for hemothorax during 72 hours after catheterization suffered massive hemothorax, developing immediately after removal of the catheter and requiring thoracotomy.
After central venous catheterization, chest radiography is mandatory to confirm the proper location of catheter tip. Normally, catheters should be parallel to the wall of central veins and show a gentle curvature within the superior vena cava [11]. In this case, however, we did not find any evidence for injury of superior vena cava on chest X-ray (Fig. 1).

The success rate of subclavian vein catheterization by anatomical landmark technique is associated with the experience of clinicians. Also, fatal complications such as hemothorax, hemomediastinum and cardiac tamponade can occur by repeated attempts and accidental puncture of arteries. Therefore, ultrasound guided central venous cannulation can be very useful in speed and patients' safety and comfort, especially in patients where there is difficult access despite repeated attempts. Reductions in the failure rate from $55 \%$ to $8 \%$ and the complication rate from $41 \%$ to $4 \%$ was reported with the use of ultrasound in subclavian vein catheterization compared to anatomic landmark-based techniques [12]. Hence, we suggest that physicians should be familiar with ultrasound-guided technique through adequate training.

In conclusion, the authors re-emphasize careful monitoring even after subclavian venous catheter removal to detect this kind of potentially fatal complication and the need for ultrasound guidance during insertion of central venous catheters.

\section{References}

1. McGee DC, Gould MK. Preventing complications of central venous catheterization. N Engl J Med 2003; 348: 1123-33.

2. Sidiropoulou Z, Joao P, Vasconcelos P, Couceiro C. Right subclavian vein catheterism complication due to a 'foreign body': a case report. J Med Case Reports 2010; 4: 327.

3. Mansfield PF, Hohn DC, Fornage BD, Gregurich MA, Ota DM. Complications and failures of subclavian-vein catheterization. N Engl J Med 1994; 331: 1735-8.

4. Holt S, Kirkham N, Myerscough E. Haemothorax after subclavian vein cannulation. Thorax 1977; 32: 101-3.

5. Nordback I, Baer G. Massive hemothorax in a child after removal of subclavian vein catheter. Acta Chir Scand 1982; 148: 701-2.

6. Collini A, Nepi S, Ruggieri G, Carmellini M. Massive hemothorax after removal of subclavian vein catheter: a very unusual complication. Crit Care Med 2002; 30: 697-8.

7. Ellis LM, Vogel SB, Copeland EM 3rd. Central venous catheter vascular erosions. Diagnosis and clinical course. Ann Surg 1989; 209: 475-8.

8. Duntley P, Siever J, Korwes ML, Harpel K, Heffner JE. Vascular erosion by central venous catheters. Clinical features and outcome. Chest 1992; 101: 1633-8.

9. Jay AW, Aldridge HE. Perforation of the heart or vena cava by central venous catheters inserted for monitoring or infusion therapy. CMAJ 1986; 135: 1143-4.

10. Sturm JT, Marsh DG, Bodily KC. Ruptured thoracic aorta: evolving radiological concepts. Surgery 1979; 85: 363-7.

11. Skolnick ML. The role of sonography in the placement and management of jugular and subclavian central venous catheters. AJR Am J Roentgenol 1994; 163: 291-5.

12. Randolph AG, Cook DJ, Gonzales CA, Pribble CG. Ultrasound guidance for placement of central venous catheters: a meta-analysis of the literature. Crit Care Med 1996; 24: 2053-8. 